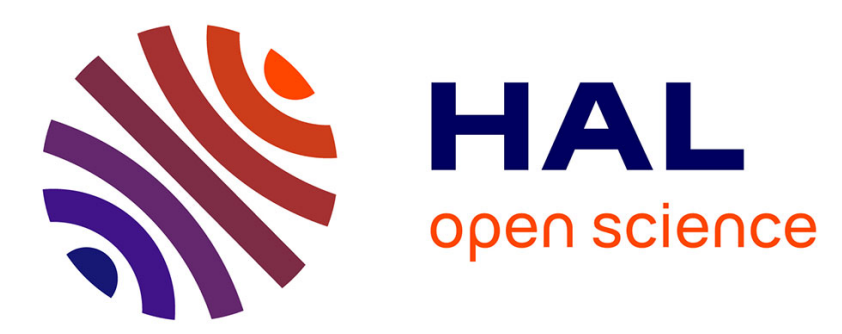

\title{
Nonlinear interaction between energetic particles and turbulence in gyro-kinetic simulations and impact on turbulence properties
}

\author{
David Zarzoso, P. Migliano, V. Grandgirard, G. Latu, C. Passeron
}

\section{To cite this version:}

David Zarzoso, P. Migliano, V. Grandgirard, G. Latu, C. Passeron. Nonlinear interaction between energetic particles and turbulence in gyro-kinetic simulations and impact on turbulence properties. Nuclear Fusion, 2017, 57 (7), 10.1088/1741-4326/aa7351 . hal-01791621

\section{HAL Id: hal-01791621 \\ https://hal.science/hal-01791621}

Submitted on 14 May 2018

HAL is a multi-disciplinary open access archive for the deposit and dissemination of scientific research documents, whether they are published or not. The documents may come from teaching and research institutions in France or abroad, or from public or private research centers.
L'archive ouverte pluridisciplinaire $\mathbf{H A L}$, est destinée au dépôt et à la diffusion de documents scientifiques de niveau recherche, publiés ou non, émanant des établissements d'enseignement et de recherche français ou étrangers, des laboratoires publics ou privés. 


\title{
Nonlinear interaction between energetic particles and turbulence in gyro-kinetic simulations and impact on turbulence properties
}

\author{
D. Zarzoso* and P. Migliano \\ Aix-Marseille Université, CNRS, PIIM, UMR 7345 Marseille, France. \\ V. Grandgirard, G. Latu, C. Passeron \\ CEA, IRFM, 13108 Saint-Paul-lez-Durance, France.
}

The modification of radial structure, frequency and intensity of turbulent transport in the presence of energetic-particle-driven geodesic acoustic modes (EGAMs) is analysed by means of full- $f$ global gyro-kinetic simulations using GYSELA code. It is observed that turbulence leads to a smoother evolution of the distribution function, less pronounced flattening of the distribution function in velocity space during the nonlinear saturation of EGAMs and reduced saturation level of electrostatic potential with respect to the case where turbulence is artificially suppressed. It is shown that EGAMs are excited and impact turbulent transport in the region where the EP is localised, fading away the staircase structure observed in the absence of energetic particles. For the first time, evidences of a three-wave coupling between turbulent modes and EGAMs in gyro-kinetic simulations are provided by means of bispectral analysis using wavelet transform in time. The coupling evolves from the standard self-regulation of turbulence by the zero-frequency zonal component to a steady-state regime where turbulence dynamics is dominated by the zonal component oscillating at the EGAM frequency.

* david.zarzoso-fernandez@polytechnique.org 


\section{INTRODUCTION}

Turbulent transport is one of the main issues to be understood, predicted and controlled, to achieve steady-state operation in future nuclear fusion devices such as ITER. In addition to turbulence, in present devices additional heating such as Neutral Beam Injection (NBI) and Ion Cyclotron Resonance Heating (ICRH) leads to the formation of long tails in the distribution function, which indicates the presence of energetic particles (EP), having energies larger than those of thermal particles. EP will also be present in future nuclear reactors, where an important population of $\alpha$ particles will be produced during the fusion reactions. References [1,2] are recent reviews on EP and EP modes with appropriate references to previous work on this topic. Energetic particles must be sufficiently well confined in order to transfer their energy, via Coulomb collisions, to thermal particles and sustain this way the nuclear fusion reactions. However, energetic particles excite EP modes. This excitation is due to the inversion of the slope of the distribution function in phase space, i.e. either in real space or in energy space (parallel and/or perpendicular velocity). In the present paper we focus our analysis on the excitation and nonlinear saturation of a class of EP modes called energetic geodesic acoustic modes (EGAMs), excited by non uniformities of the distribution function in velocity space. EGAMs were predicted theoretically [3-8], observed experimentally [9-12] and analysed in numerical simulations [6, 13-16]. As the standard GAMs [17], they are characterised by axisymmetric components of electrostatic potential, density and pressure. The electrostatic potential is dominated by a zonal structure, whereas density and pressure exhibit a poloidally up-down asymmetric structure, which is also visible in the electrostatic potential. Because of the axisymmetry of EGAMs, they have been thought to contribute little to the transport of particles and energy, but to play a role on the stabilisation of turbulence, as was analysed for the standard GAM [18] and observed in experiments in the AUG tokamak [19], similar to what is believed for the zonal flow [20]. However, EGAMs might have a negative impact on the particle confinement as suggested by measurements reported in [11]. In addition, they have been found to modify nonlocally turbulent transport, for instance when fronts propagate outwards (as observed in ITG turbulence) and couple to static oscillations of radial profiles (characteristic of EGAMs) [13]. Because of the importance of both turbulence and EP and because they naturally co-exist in

present and future fusion devices, further studies regarding the modification of turbulent transport 
by EP are needed to characterise the way EP modes and turbulent modes interact. In the present paper, we investigate the interaction between turbulence and EGAMs when varying the amplitude of the latter and show evidences of a local coupling between EGAMs and turbulent modes by means of bispectral analysis. The remainder of the paper is structured as follows. In section II we describe the Gysela code, the simulations that we have performed and the initial turbulent state where we introduce energetic particles. In section III we present the linear excitation of EGAMs and provide the reader with first evidences of the role of turbulence on the saturation of the EP mode. In section IV it is analysed in detail the radial structure of the EGAM and the modification of turbulent intensity and transport in the presence of EGAMs. Section V provides the first evidence of local coupling between EGAMs and turbulent modes in gyro-kinetic simulations by means of bispectral analysis using wavelet transform in time. Conclusions and future work are presented in section VI.

\section{DESCRIPTION OF GYSELA AND THE SIMULATIONS}

The simulations are performed with the full- $f$, global, flux-driven gyro-kinetic code GySELA [21]. The simulations are similar to those reported in Ref. [13]. For the sake of clarity and convenience, we give again in this section a brief description of the GYSELA code and the simulations that we perform to obtain the results presented in this paper.

\section{A. Description of GYSELA}

All quantities in Gysela are dimensionless, using three physical parameters that one can use to calculate the dimensional physical quantities. Those three physical parameters are temperature $T_{0}$ (in $\mathrm{eV}$ ), density $n_{0}\left(\right.$ in $\left.\mathrm{m}^{-3}\right)$ and magnetic field $B_{0}$ (in $\mathrm{T}$ ). Using these three parameters, one can calculate the thermal velocity $v_{\text {th }}=\sqrt{T_{0} / m_{i}}$, where $m_{i}$ is the ion mass, the ion cyclotron frequency $\omega_{c}=e B_{0} / m_{i}$, where $e$ is the elementary charge (we assume atomic number $Z=1$ ), and the ion Larmor radius $\rho_{i}=v_{\mathrm{th}} / \omega_{c}$. Lengths are normalised to $\rho_{i}$, time is normalised to $\omega_{c}^{-1}$, velocities are normalised to $v_{\mathrm{th}}$, the ion guiding-centre distribution function is normalised to $n_{0} / v_{\mathrm{th}}^{3}$ and the electrostatic potential is normalised to $T_{0} / e$. With these normalisations, GysELA solves the 
dimensionless gyrokinetic equations for the full- $f$ ion guiding-centre distribution function within the modern gyrokinetic formalism [22] in the electrostatic limit using adiabatic electrons

$$
\begin{gathered}
\partial_{t} F+\frac{1}{B_{\|}^{*}} \nabla \cdot\left(B_{\|}^{*} \dot{\mathbf{x}}_{G} F\right)+\frac{1}{B_{\|}^{*}} \partial_{v_{G \|}}\left(B_{\|}^{*} \dot{v}_{G \|} F\right)=\mathcal{C}(F)+\mathcal{D}(F)+S_{\mathrm{th}}+S_{\mathrm{EP}} \\
\dot{\mathbf{x}}_{G}=v_{G \|} \mathbf{B}^{*}+\mathbf{b} \times \nabla \Xi \\
\dot{v}_{G \|}=-\mathbf{B}^{*} \cdot \nabla \Xi \\
\tau(\phi-\langle\phi\rangle)-\frac{1}{n_{e q}} \nabla_{\perp} \cdot\left(n_{e q} \nabla_{\perp} \phi\right)=\frac{n_{G}-n_{G, e q}}{n_{e q}}
\end{gathered}
$$

with $\tau=T_{i} / T_{e},\langle\cdot\rangle$ the flux-surface average, $\nabla \Xi=\mu \nabla B+\nabla J_{0} \cdot \phi, \mu$ is the magnetic moment, $\mathbf{B}^{*}=\mathbf{B}+\left(m_{i} / e\right) v_{G \|} \nabla \times \mathbf{b}, J_{0}$ is the gyro-average operator, $B_{\|}^{*}=\mathbf{B}^{*} \cdot \mathbf{b}$ and $\mathbf{b}=\mathbf{B} / B$, with $B$ the modulus of the magnetic field vector. The ion guiding-centre density is defined as $n_{G}=$ $\int \mathcal{J}_{v} \mathrm{~d} \mu \mathrm{d} v_{G \|}\left(J_{0} \cdot F\right)$ with $\mathcal{J}_{v}$ the Jacobian in velocity space. When calculating the equilibrium guiding-centre density $n_{G, e q}$ the guiding-centre distribution function $F$ is replaced by the guidingcentre equilibrium distribution function $F_{\text {eq }}$, defined as the flux-surface average of the guidingcentre distribution function. The magnetic topology consists of concentric toroidal flux surfaces with circular poloidal cross-sections without Shafranov shift. The magnetic field is defined as $\mathbf{B}=\left(B_{0} R_{0} / R\right)\left[\zeta(r) \mathbf{e}_{\theta}+\mathbf{e}_{\varphi}\right]$, with $\zeta(r)=r /\left(q R_{0}\right)$. The quantities $B_{0}$ and $R_{0}$ are, respectively, the magnetic field and the major radius of the tokamak evaluated at the magnetic axis. The vectors $\mathbf{e}_{\theta}=r \nabla \theta$ and $\mathbf{e}_{\varphi}=R \nabla \varphi$ are the unit vectors in the poloidal and toroidal directions, respectively, with $r$ the minor radius and $R$ the major radius of the tokamak. The angles $\theta$ and $\varphi$ are the poloidal and toroidal angles, respectively. The safety factor $q$ is defined as $q(r)=q_{1}+q_{2}(r / a)^{q_{3}}$, with $a$ the minor radius of the poloidal cross-section, defining the size of the tokamak.

The collision operator is a simplified Lorentz-type operator in parallel velocity space

$$
\mathcal{C}(F)=\frac{1}{B_{\|}^{*}} \frac{\partial}{\partial v_{G \|}}\left\{B_{\|}^{*}\left(\mathcal{D}_{\|} \frac{\partial F}{\partial v_{G \|}}-\nu_{\|} F\right)\right\}
$$

where the coefficients $\mathcal{D}_{\|}$and $\nu_{\|}$model, respectively, a diffusion and a drag in the parallel velocity direction. By conveniently defining the local fluid velocity and ion temperature of the shifted Maxwellian $F_{M}$ satisfying $\mathcal{C}\left(F_{M}\right)=0$, the collision operator ensures energy and parallel momentum conservation. 
To avoid strong gradients at the boundaries, radial diffusion is applied in the inner and outer buffer regions by means of the operator

$$
\mathcal{D}(F)=\frac{1}{B_{\|}^{*}}\left[\frac{1}{r} \frac{\partial}{\partial r}\left(r \chi_{0} H_{\text {buff }}(r) B_{\|}^{*} \frac{\partial}{\partial r} F\right)\right]
$$

where $\chi_{0}$ is the diffusion coefficient and

$$
H_{\text {buff }}(r)=1+\frac{1}{2}\left[\tanh \left(\frac{r-r_{\max }+B_{L} L_{r}}{B_{S} L_{r}}\right)-\tanh \left(\frac{r-r_{\max }-B_{L} L_{r}}{B_{S} L_{r}}\right)\right]
$$

approximates a step-like function, where $L_{r}$ is the length of the radial domain and $B_{L}$ and $B_{S}$ are the location and the stiffness, respectively, of the buffer region. The buffer region represents a sink for energy.

Two sources are used for the results reported in the present paper: a source of thermal energy $\left(S_{\text {th }}\right)$ to maintain the radial profiles well above the threshold for the excitation of ITG modes and an energetic particle source $\left(S_{\mathrm{EP}}\right)$ that accelerates particles in the parallel direction eventually providing an inversion of the slope of the distribution function in parallel velocity space. The mathematical expressions of these two sources are given by projecting onto Hermite and Laguerre polynomial bases

$$
\begin{gathered}
S_{\mathrm{th}}=S_{\mathrm{th}, 0}(t) S_{\mathrm{th}, \mathrm{r}}(r) \sum_{h, l} c_{h l}^{\mathrm{th}} \mathcal{H}_{h}\left(v_{G \|}\right) \mathcal{L}(\mu B(r, \theta)) \mathrm{e}^{-\frac{v_{G} \|^{2}}{2 T_{\mathrm{th}}}} \mathrm{e}^{-\frac{\mu B(r, \theta)}{T_{\mathrm{th}}}} \\
S_{\mathrm{EP}}=S_{\mathrm{EP}, 0}(t) S_{\mathrm{EP}, \mathrm{r}}(r)\left(S_{+}+S_{-}\right)
\end{gathered}
$$

where $S_{\mathrm{th}, 0}(t)$ and $S_{\mathrm{EP}, 0}(t)$ are the amplitudes of thermal and energetic particle sources, respectively, $S_{\mathrm{th}, \mathrm{r}}(r)$ and $S_{\mathrm{EP}, \mathrm{r}}(r)$ are their respective radial envelopes and

$$
S_{ \pm}=\sum_{h, l} c_{h l}^{\mathrm{EP}} \mathcal{H}_{h}\left(v_{G \|} \pm v_{0}\right) \mathcal{L}(\mu B(r, \theta)) \mathrm{e}^{-\frac{\left(v_{G \|} \pm v_{0}\right)^{2}}{2 T_{\mathrm{EP},} \|}} \mathrm{e}^{-\frac{\mu B(r, \theta)}{T_{\mathrm{EP},} \perp}}
$$

The coefficients $c_{h l}^{\text {th }}$ and $c_{h l}^{\mathrm{EP}}$ are chosen in such a way that $S_{\text {th }}$ and $S_{\mathrm{EP}}$ provide pure energy injection. The particular choice for $S_{\mathrm{EP}}$ ensures that the equilibrium distribution function remains proportional to $\exp (-\mu B / T)$, i.e. there is no shift in $v_{\perp}$. Taking into account that the quasi-neutrality condition 4 is obtained assuming that the distribution function is a Maxwellian in perpendicular velocity space, no modification of the quasi-neutrality condition is needed for our 


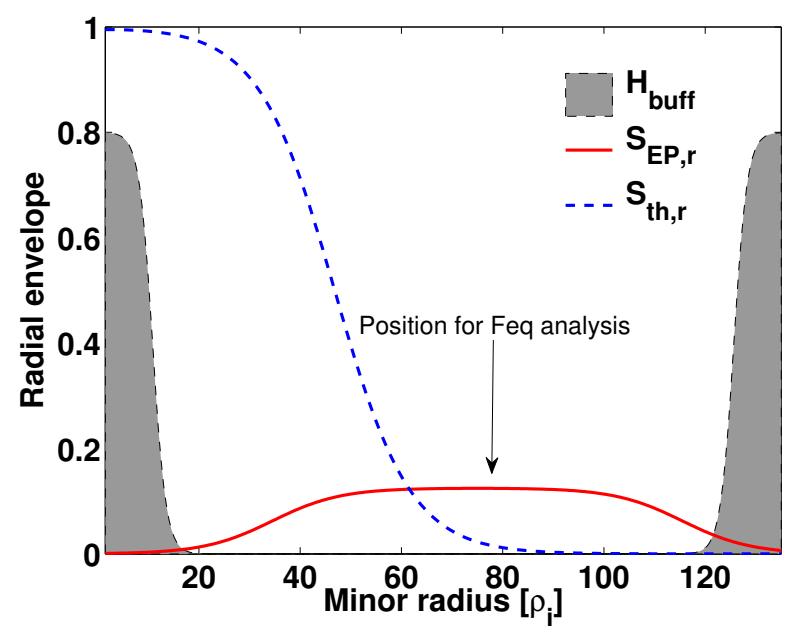

FIG. 1: Radial envelope of thermal source (dashed blue line), energetic particle source (red line) and buffer region (shadowed area). The amplitudes are arbitrary.

simulations. This choice represents therefore a compromise to excite EGAMs without major modifications of the quasi-neutrality solver. The calculation of $c_{h l}^{\text {th }}$ under the constraint of pure energy injection gives the following thermal source

$$
S_{\mathrm{th}}=\left[v_{G \|^{2}}-\frac{1}{2}-\frac{J_{\| B}}{2-J_{\| B}^{2}}\left(2-\frac{\mu B}{T_{\mathrm{th}}}\right)\left(2 v_{G \|}-J_{\| B}\right)\right] \mathrm{e}^{-\frac{v_{G} \|^{2}}{2 T_{\mathrm{th}}}} \mathrm{e}^{-\frac{\mu B}{T_{\mathrm{th}}}}
$$

where $J_{\| B}=\sqrt{2 m T_{\mathrm{th}}} /\left(e Z B^{2}\right) \mu_{0} J_{\|}$, with $\mu_{0}$ the permeability constant and $J_{\|}$the equilibrium parallel current.

Similarly, the calculation of $c_{h l}^{\mathrm{EP}}$ under the constraint of pure energy injection gives the following

$$
\begin{array}{r}
S_{ \pm}=\left[\left(v_{G \|} \pm v_{0}\right)^{2}-\frac{1}{2}-\frac{J_{\| B}}{2-J_{\| B}^{2}\left(1+2 v_{0}^{2}\right)}\left(2-\frac{\mu B}{T_{\perp}}\right)\left(2\left(v_{G \|} \pm v_{0}\right)-J_{\| B}\right)\right] \times \\
\mathrm{e}^{-\frac{\left(v_{G \|} \pm v_{0}\right)^{2}}{2 T_{\mathrm{EP}, \|}}} \mathrm{e}^{-\frac{\mu B}{T_{\mathrm{EP}, \perp}}}
\end{array}
$$

The radial envelope of the thermal and energetic particle sources together with the radial envelope of the buffer region are given in figure 1 . The blue line represents the thermal source and the red line the energetic particle source. The shadowed area indicates the buffer region. Indicated is also the radial position where the analysis of the equilibrium distribution function is done in the following. An example of the dependence of the sources on the parallel velocity is given in figure 2 . 


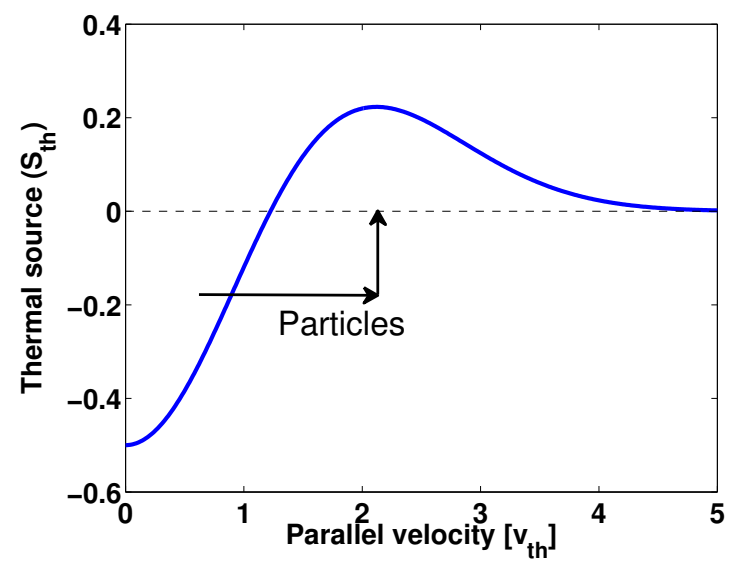

(a)

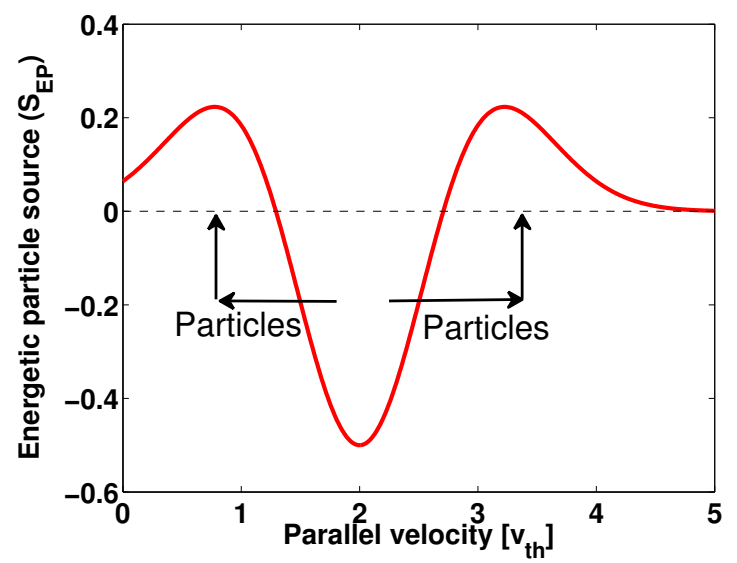

(b)

FIG. 2: Schematic view of the thermal (left) and energetic particle (right) sources as a function of the velocity and parallel velocity, respectively.

It can be observed that the thermal source accelerates particles around a velocity $v \approx 0$, whereas the energetic particle source accelerates and decelerates particles around a velocity $v_{0} \approx 2$.

The parameters $v_{0}$ and $T_{\mathrm{EP}, \|}$ are critical for the inversion of the slope of the equilibrium distribution function. However, the version of $S_{\mathrm{EP}}$ used in this paper exhibits a limitation regarding the interval for $v_{0}$. This is due to the fact that the source is not an operator applied on the distribution function and it is therefore always acting independently of the value of the distribution function. As a consequence, if $v_{0}$ is large enough, the distribution function can exhibit negative values.

The EP source used in this simulation, although not realistic, tries to mimic the generation of energetic particles, making the system evolve towards a state where the slope of the distribution function is positive in a region of phase space. Therefore, the EP source cannot be used to predict the direct impact of external heating on confinement or turbulence properties, but only as a means to introduce energetic particles in the system and analyse their nonlinear interaction with background turbulent modes. Note also that in the simulations the presence of the collision operator is essential to balance the source of energetic particles. The system must therefore be understood as a very complex physical system in five dimensions with injection of energy that is distributed in phase space via collisions and transported in real space via neoclassical and turbulent transport. Part of 
this energy will be used to excite EGAMs, that can eventually interact with turbulence, modifying this way the transport. The system is closed by the presence of sinks of energy localised at the buffer regions.

\section{B. Description of the simulations and the initial turbulent state}

In the present paper, we report only on simulations where energetic particles are introduced by means of the source given by expression 9. We use Neumann (resp. Dirichlet) boundary conditions for the inner (resp. outer) radial position. The resolution is $\left(N_{r}, N_{\theta}, N_{\varphi}, N_{v_{\|}}, N_{\mu}\right)=$ $(256,256,128,128,32)$. This resolution represents a good compromise to to describe both ITG modes and EGAMs from the point of view of the spatial and velocity structure optimising the use of CPU time. In particular, the resolution for ITG turbulence simulations in GysELA has been thouroughly checked in the past in similar simulations for the study of ITG turbulence [21]. As far as EGAMs are concerned, it has been shown that gyro-kinetic simulations with similar numerical resolution in phase space are sufficient to reproduce with excellent agreement theoretical predictions for the linear growth rate and frequency [7]. Convergence tests have also shown that the radial structure of EGAM is well described with the resolution used in this paper [15]. Regarding the nonlinear saturation of the EGAM, we focus our efforts on the interaction with ITG modes. Therefore we do not aim at describing the formation of structures in phase space, smaller than the main island in $\left(\theta, v_{\|}\right)$, and leading for example to the chirping behaviour. These small structures would require increased resolution in parallel velocity space (more than 2000 points), which would dramatically increase the computational cost making these simulations prohibited. The ion Larmor radius normalised to the minor radius of the tokamak is $\rho_{*}=1 / 150$ and the ratio between ion and electron temperatures is $\tau=1$. The radial extension of the simulation box is $10^{-2} \leq r / a \leq 0.9$, which is equivalent to 135 ion Larmor radii. The inverse aspect ratio giving the ratio between the major radius at the magnetic axis and the minor radius of the poloidal cross section is $R_{0} / a=3.2$. The safety factor is defined by the parameters $q_{1}=2.5, q_{2}=0.7$ and $q_{3}=2.0$. The initial density and temperature profiles are defined by its normalised logarithmic gradients $R / L_{T} \equiv R_{0} T^{-1} \mathrm{~d} T / d r=6.5$ and $R / L_{n} \equiv R_{0} n^{-1} \mathrm{~d} n / d r=2.2$, with the temperature calculated as $T=p / n$, where $p$ is the kinetic pressure. Note that for our simulations the choice of 


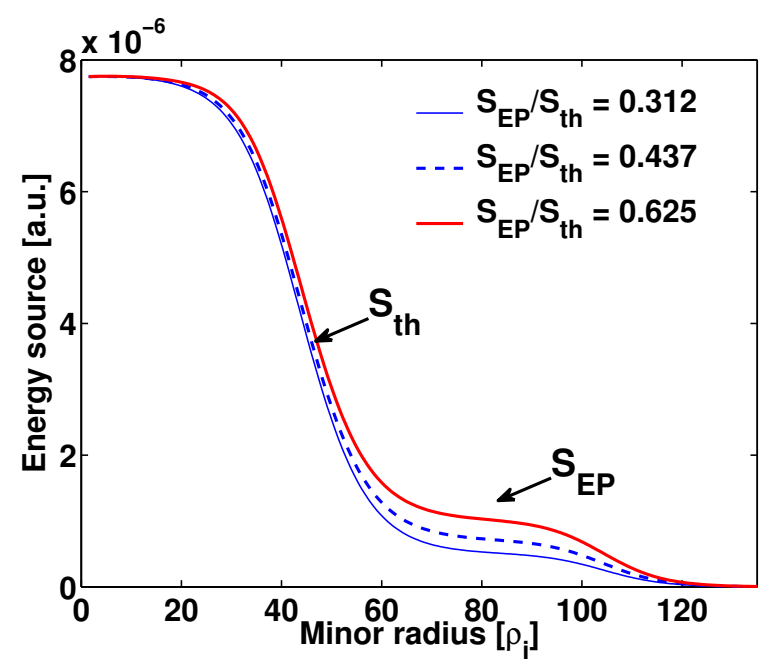

FIG. 3: Sum of the thermal and energetic particle sources for the three simulations presented in this paper. The main peak in the inner radial region is due to the presence of the source injecting only thermal energy. The tail in the mid and outer radial positions is due to the presence of the source injecting suprathermal energy.

$R / L_{T}$ is well beyond the linear threshold $R / L_{T, \text { threshold }} \approx 3$, as calculated from linear gyro-kinetic simulations in agreement with linear gyro-kinetic theory [? ]. All our simulations are run in the banana regime, with a collisionality $\nu_{*}=0.02$.

The thermal source is located around the minimum radial position $r=0$. The EP source is located around the mid radial position, with quite a large radial extension in order to avoid any localised modification of radial profiles that might have a direct impact on turbulence. The parameter $v_{0}=2$ and the perpendicular and parallel temperatures of the EP source satisfy $T_{\mathrm{EP}, \perp}=$ $2 T_{\mathrm{EP}, \|}$. Three simulations are presented, the only difference being the amplitude of the EP source. The scan proposed in this paper covers the amplitudes $S_{\mathrm{EP}} / S_{\mathrm{th}} \in[0.312,0.437,0.625]$. The radial profile of the sum of the thermal and energetic particle sources is represented in figure 3 for the three simulations considered in the paper.

The simulations are run first with the thermal source $S_{\text {th }}$ but without the EP source $S_{\mathrm{EP}}$, to reach a statistically turbulent steady state, up to $\omega_{c} t_{0} \approx 9 \cdot 10^{5}$. On the left panel of figure 4 we give the radial profiles of the guiding-centre temperature and density, together with the radial envelope of the buffer region. Both temperature and density are averaged over a time window $\omega_{c} \Delta t \approx 3 \cdot 10^{4}$. 
On the right panel of figure 4 we plot the radial profile of the turbulent intensity in the stastistically turbulent steady-state, calculated as follows

$$
I_{\text {turb }}(r)=\frac{1}{\Delta t} \int_{\Delta t} \mathrm{~d} t\left(\sum_{m>1}\left|\hat{\phi}_{m}(r, \varphi=0, t)\right|^{2}\right)^{1 / 2}
$$

where $\Delta t$ is the width of the time window $\left(\omega_{c} \Delta t \approx 3 \cdot 10^{4}\right)$ where turbulent intensity is calculated, and $\hat{\phi}_{m}$ is the Fourier mode in the poloidal direction evaluated at the fixed toroidal position $\varphi=0$. Since we are considering a fixed toroidal position, we are considering also the axisymmetric modes. Ideally one should use the Fourier mode $\hat{\phi}_{m n}$ for $n>0$ in both poloidal and toroidal directions. However, due to numerical and memory constraints, one cannot have a sufficiently well time resolved 3D diagnostic and must use the electrostatic potential at a given toroidal position for radial profiles. Although expression 13 is an approximation for the turbulent intensity, the resonant condition of ITG modes $q \approx m / n$, ensures that the axisymmetric modes $n=0$ do not contribute significantly. The only axisymmetric components that could enter in the expression are related to higher poloidal harmonic GAMs. Since the GAM amplitude decreases with the poloidal mode number $m$, the main components of GAMs are $m=0$ and $m=1$ and therefore GAMs do not contribute significantly in expression 13.

The right panel of figure 4 is of particular interest, since the radial pattern of the turbulent intensity is reminiscent of self-organised flow structure called $E \times B$ staircase, already observed in previous GySELA simulations [23] and more recently identified in Tore Supra tokamak by means of high-resolution fast-sweeping X-mode reflectometry [24]. This staircase structure is characterised by regions with increased turbulence, the separation between those regions being of the order of $20-30 \rho_{i}[24]$.

\section{ONSET OF THE ENERGETIC PARTICLE SOURCE: INITIAL REDUCTION OF TURBULENCE, EXCITATION AND NONLINEAR SATURATION OF EGAMS IN THE PRESENCE OF TURBULENCE}

Once the statistically turbulent steady-state is reached, the simulation is split into three simulations, where the EP source is switched on and the amplitude changed for each simulation. Therefore, all the simulations are re-started from the same turbulent regime described in the previous section. 


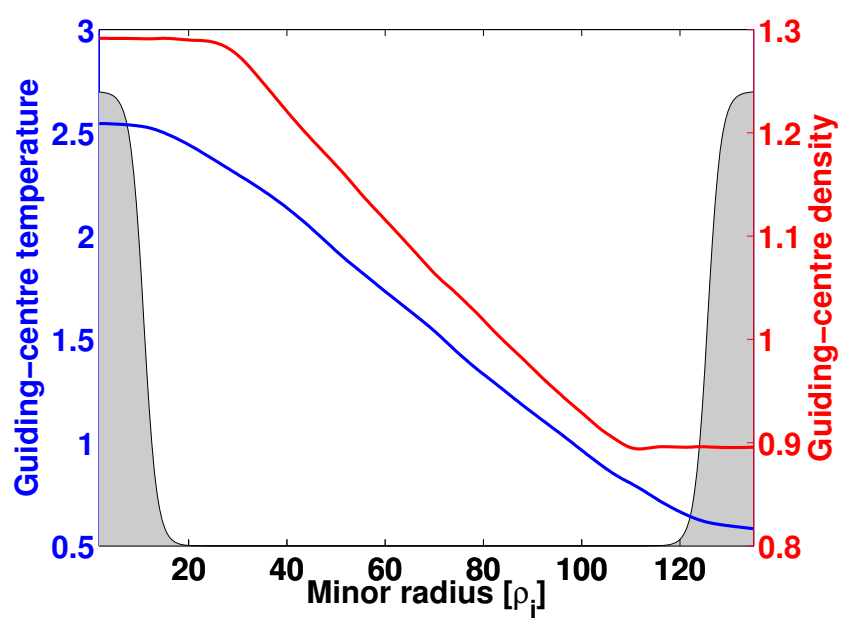

(a)

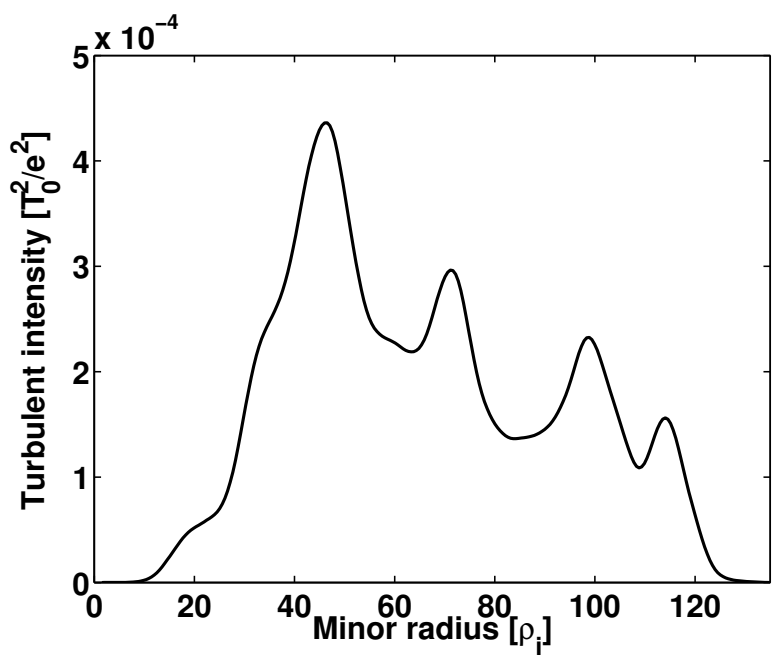

(b)

FIG. 4: (Left) Radial profiles of ion guiding-centre temperature (blue line) and density (red line). The shadowed region represents the radial envelope of the buffer region in arbitrary units. (Right) Radial profile of the turbulent intensity as calculated by expression 13, averaged over

$$
\omega_{c} \Delta t \approx 3 \cdot 10^{4} .
$$

After switching on the EP source, the simulations are run long enough (up to $\omega_{c} t \approx 1.3 \cdot 10^{6}$ ) to reach a new statistically steady state, where both ITG modes and EGAMs coexist in nonlinear regime.

Much effort has been done in the past to develop the analytic theory of EGAMs [3, 4, 7, 8] and the interested reader will be able to find further details in the previous litterature. Nevertheless, for the sake of completeness and clarity we provide hereafter the reader with a simplified model to understand the basic features of the instability, to be used in the following, namely the resonant velocity and the drive of the instability. We can write a simplified gyro-kinetic Vlasov equation in the long-wavelength approximation $\left(J_{0} \rightarrow 1\right)$ neglecting the curvature terms and the $E \times B$ drift as

$$
\partial_{t} F+v_{G \|} \nabla_{\|} F+\frac{e \cdot E_{\|}}{m v_{G \|}} \partial_{v_{G \|}} F=0
$$

where $E_{\|}$is the parallel electric field. We decompose the distribution function into equilibrium and perturbed distribution functions $F=F_{\text {eq }}+\delta F$, where by definition $F_{\text {eq }}$ depends only on the motion 
invariants, i.e. $F_{\text {eq }}=F_{\text {eq }}\left(E, \mu, P_{\varphi}\right.$, ), with $E$ the energy and $P_{\varphi}$ the toroidal momentum, which can be related to the radial position. Therefore, the equilibrium distribution function is obtained, as in GySELA, as a flux-surface average of the guiding-centre distribution function

$$
F_{\text {eq }}=\frac{1}{4 \pi^{2}} \int \mathrm{d} \theta \mathrm{d} \varphi F
$$

Linearising equation 14 and performing Fourier transforms in time and parallel direction, we obtain the linear response

$$
\delta \hat{F}=-\frac{i 2 e k_{\|} \hat{\phi}}{\omega-k_{\|} v_{G \|}} \frac{\partial F_{\mathrm{eq}}}{\partial E}
$$

with $k_{\|}=1 /\left(q R_{0}\right)$. From equation 16 it is clear that the resonant velocity turns out to be $v_{\text {res }}=q R_{0} \omega$. The time evolution of the kinetic energy of particles is given by the product $\mathbf{J} \cdot \mathbf{E}$, with $\mathbf{J}$ the perturbed parallel current. Owing to the energy conservation, the time evolution of the energy of the mode is

$$
\frac{\mathrm{d} \mathcal{E}}{\mathrm{d} t}=-i \int \mathrm{d} v_{G \|} \frac{2 e\left|k_{\|} \hat{\phi}\right|^{2}}{\omega-k_{\|} v_{G \|}} \frac{\partial F_{\mathrm{eq}}}{\partial E}
$$

which can be rewritten using the Dirac distribution as follows

$$
\frac{\mathrm{d} \mathcal{E}}{\mathrm{d} t}=e 2 \pi\left|k_{\|} \hat{\phi}\right|^{2} \int \mathrm{d} v_{G \|} \delta\left(\omega-k_{\|} v_{G \|}\right) \frac{\partial F_{\mathrm{eq}}}{\partial E}=\left.e 2 \pi\left|k_{\|}\right||\hat{\phi}|^{2} \frac{\partial F_{\mathrm{eq}}}{\partial E}\right|_{v_{G \|}= \pm v_{\mathrm{res}}}
$$

From equation 18 we can see that the derivative of the equilibrium distribution function with respect to the energy at the resonant velocity is the drive of the instability. Therefore, for the analysis of the drive of the EGAM computing the slope of the equilibrium distribution function in velocity space evaluated at the resonant velocity is mandatory.

As explained in Ref. [13], the first effect of the EP source is to accelerate particles from thermal to suprathermal parallel velocities. This leads to a depletion of the distribution function around the thermal velocity, where particles resonate with ITG modes. The depletion results in a stabilisation of ITG modes and therefore in a reduction of turbulent transport. This depletion is clearly observed in figure 5, where we plot the time trace of the integral in perpendicular velocity of the equilibrium distribution at the velocity $v_{0}$ where the EP source is taking particles to accelerate/decelerate them. In that figure the distribution function is normalised to its value just before the instant where the EP source is applied. The colours represent the different simulations performed, varying the amplitude of the EP source. The depletion occurs roughly up to $\omega_{c} t \approx 1.1 \cdot 10^{6}$. What happens 


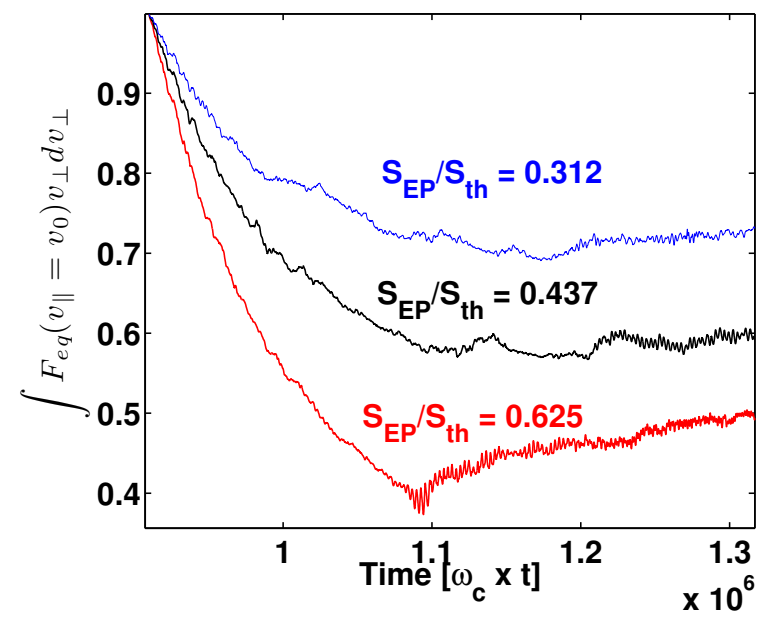

FIG. 5: Time evolution of the integral in perpendicular velocity equilibrium distribution function at the parallel velocity $v_{0}$ where the EP source is taking particles for the three simulations used in this paper. The distribution function is normalised its value just before the introduction of the EP source.

after that time will be analysed in the following, in particular the excitation of EGAMs and the subsequent evolution of turbulence. The common behaviour of the three simulations is thus the initial reduction of turbulent intensity. Further analytic study is required regarding the linear stabilisation of ITG by energetic particles due to gradients in velocity space. However, this is well beyond the scope of the present paper, which aims at analysing the nonlinear interaction between ITG modes and EGAMs. The detailed study of linear stabilisation of ITG modes by energetic particles will therefore be left for future works. On the left panel of figure 6 we plot the radial dependence of the turbulent intensity for the three simulations used in this paper. For comparison, we give also the radial dependence of the turbulent intensity before switching on the EP source, whose radial profile is given by the shadowed region. It is clearly observed the overall decrease of turbulent intensity where the EP source is applied. On the right panel of figure 6 the time evolution of the temperature gradient averaged over the radial region $50 \rho_{i}<r<100 \rho_{i}$ is plotted. It can be observed that the reduction of turbulence is accompanied by an increase of temperature gradient within the time interval $10^{6}<\omega_{c} t<1.1 \cdot 10^{6}$.

The observed reduction is only temporary since the turbulent transport is afterwards increased 


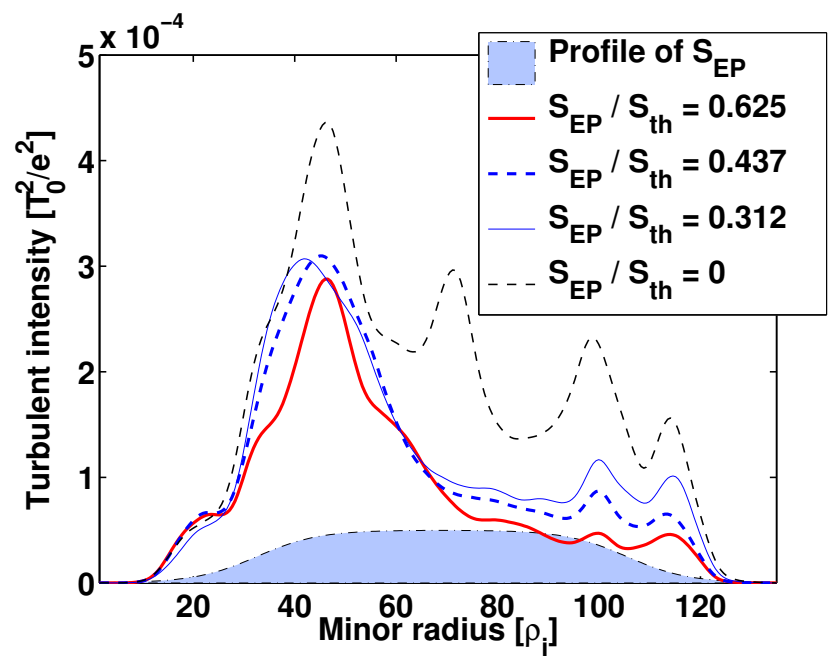

(a)

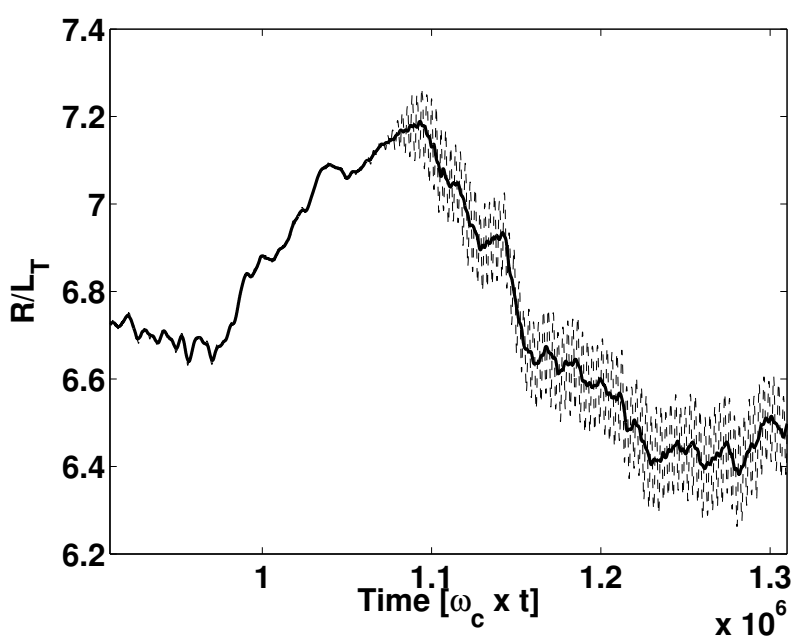

(b)

FIG. 6: (Left) Radial dependence of the turbulent intensity as given by expression 13 for the three simulations used in this paper. (Right) Time evolution of the temperature gradient after switching on the energetic particle source, averaged over the radial region $50 \rho_{i}<r<100 \rho_{i}$.

from a time $\omega_{c} t_{\text {inc }}$ that depends on the amplitude of the EP source. This can be to either a reorganisation of the system (in both real and velocity space) in the presence of the EP source, or a modification of turbulent modes when EGAMs are excited. In the remainder of this paper we investigate the possibility that EGAMs contribute to the increase of turbulent transport.

The EP source implemented in GySELA and detailed in the previous section is able to invert the slope of the distribution function. The larger the injection of parallel energy is, the larger the slope of the distribution function is. The inversion of the slope of the distribution function leads to the excitation of the EGAM[6], which can be observed when plotting the time evolution of the flux-surface averaged electrostatic potential at a given radial position (in our case we consider the mid radial position). This is shown in figure $7 \mathrm{a}$ for the three simulations used in this paper. It can be observed that the excitation occurs earlier when increasing the amplitude of the EP source, i.e. when increasing the drive of the EGAM. This is due to the fact the evolution of the slope of the equilibrium distribution function is accelerated and crosses the zero value earlier. Also, the amplitude of the electrostatic potential during the nonlinear saturation is increased with the 


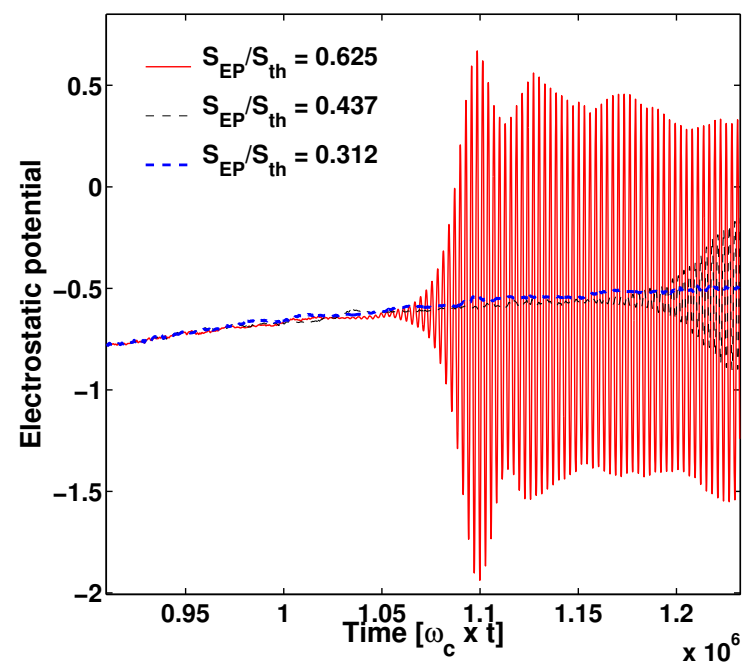

(a)

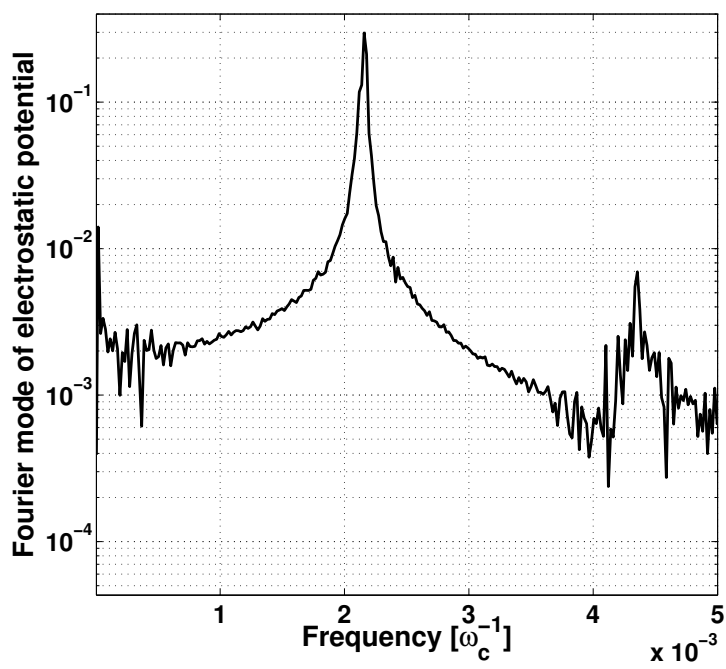

(b)

FIG. 7: (Left) Time evolution of the flux-surface averaged electrostatic potential for the three simulations. (Right) For the simulation with $S_{\mathrm{EP}} / S_{\mathrm{th}}=0.625$, time Fourier transform of the electrostatic potential after filtering out the time averaged signal.

amplitude of the EP source. Further analysis will be done in the future to analyse in detail the dependence of the amplitude of the nonlinearly saturated potential in terms of the drive in the absence of turbulence. After filtering out the time averaged signal, time Fourier transform of the flux-surface averaged electrotatic potential reveals the existence of a dominant frequency, as shown in figure $7 \mathrm{~b}$, which corresponds to the EGAM frequency $\omega_{\text {EGAM }}$. This frequency can be obtained from analytic theory, as reported in [7, 8], which has been verified against previous gyro-kinetic simulations [7]. Also benchmarking between gyro-kinetic codes [15] has revealed that this frequency is robust and depends only on the fraction of energetic particle pressure, decreasing down to roughly half the standard GAM frequency. The EGAM frequency can also be observed in figures 5 and $6 \mathrm{~b}$ from $\omega_{c} t \lesssim 1.1 \cdot 10^{6}$. Note that although the slope of the distribution function is also inverted (as we will see later) for the case $S_{\mathrm{EP}} / S_{\mathrm{th}}=0.312$, the excitation of the mode is not visible in figure 7a. This indicates the presence of additional damping mechanisms different from the Landau damping.

In figure $8 \mathrm{a}$ we plot the slope of the equilibrium distribution function, $\partial F_{\text {eq }} / \partial E$, as a function of 
time for the three amplitudes reported in the paper and evaluated at the resonant velocity, where $\omega$ is the measured frequency of the EGAM, i.e. $v_{G \|}=q R_{0} \omega_{\mathrm{EGAM}}$. Time-averaged quantities over an EGAM cycle are represented by thick lines. Also, the time-averaged over an EGAM cycle of the heat flux due to the $E \times B$ drift velocity is plotted for the sake of completeness, represented by the shadowed area and calculated as follows

$$
Q_{E \times B}=\frac{1}{4 \pi^{2}} \int \mathrm{d} \theta \mathrm{d} \varphi \int \mathcal{J}_{v} \mathrm{~d} v_{G \|} \mathrm{d} \mu \frac{1}{2} m v^{2} v_{E \times B, r}\left(J_{0} \cdot F\right)
$$

where $F$ is the guiding-centre distribution function and $v_{E \times B, r}$ is the radial component of the $E \times B$ drift velocity. For the sake of completeness, the equilibrium distribution functions without energetic particles (thin dashed line), with $S_{\mathrm{EP}} / S_{\mathrm{th}}=0.437$ (thick dashed line) and with $S_{\mathrm{EP}} / S_{\mathrm{th}}=0.625$ (solid line) just before the excitation of EGAMs are given in figure 8b, where the inversion of the slope is clearly visible for co- and counter-passing particles, leading to a bump-on-tail distribution. Note that the distribution function for $S_{\mathrm{EP}} / S_{\mathrm{th}}=0.312$ is not given in the figure for clarity. It is observed that increasing the amplitude of the EP source increases also the slope of the equilibrium distribution function and therefore the drive. Note that as mentioned earlier, the slope is inverted for the three amplitudes, even if the EGAM is only visible for two of them. Time Fourier transform of the slope of the distribution function (not shown here for the sake of clarity) reveals oscillations at the EGAM frequency. The oscillations are larger when the amplitude of the EP source is increased, in other words when the EP drive is stronger. The most interesting feature related to the evolution of the distribution function that we observe in these simulations is the evolution of the slope of the distribution function during the nonlinear saturation of the mode. The saturation of EGAM in the absence of turbulence and without radial profiles of density and pressure is due to a total flattening of the distribution function in parallel velocity space, as observed in previous gyro-kinetic simulations [6]. However, in the simulations presented in this paper, with turbulence and with radial profiles of density and pressure, we observe that the flattening is only partial. This indicates that either the presence of turbulence or the finite radial gradients result in an additional sink of energy.

To analyse the effects of radial profiles on the EGAM excitation and saturation we have performed a simulation similar to the one where $S_{\mathrm{EP}} / S_{\mathrm{th}}=0.625$, but without turbulence. This is done by filtering out every $n \neq 0$ mode after solving the quasi-neutrality equation. We have selected an amplitude of EP source so that the maximum slope of the distribution function is close to the 


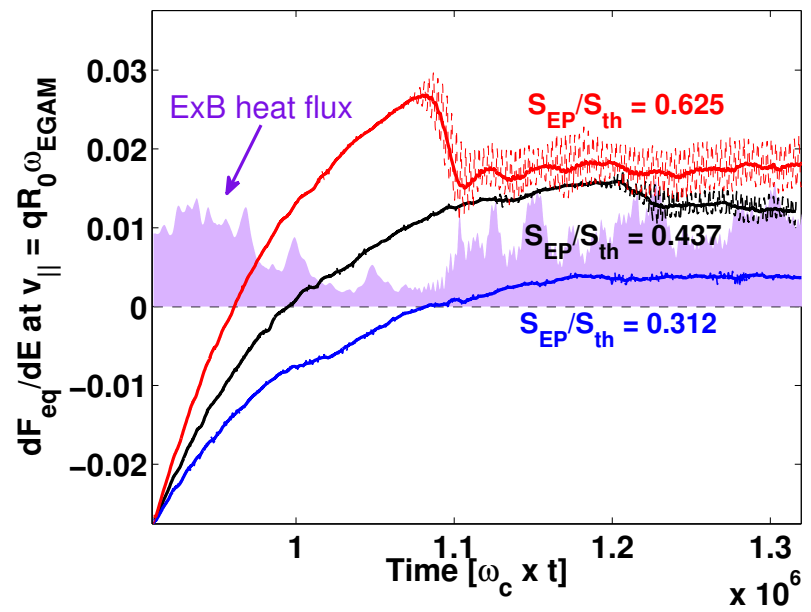

(a)

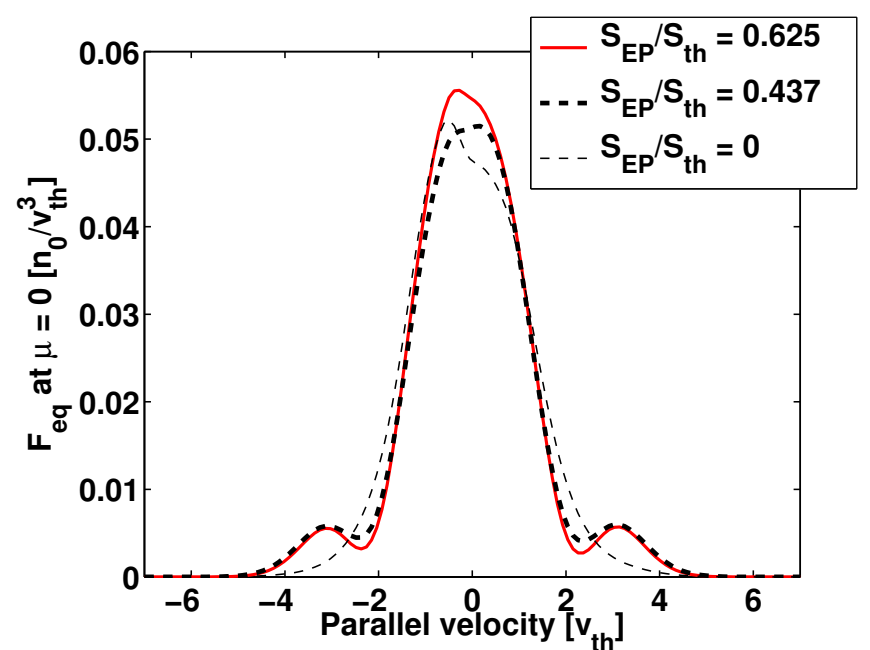

(b)

FIG. 8: (Left) Time evolution of the slope of the equilibrium distribution function (thin lines) evaluated at $v_{\|}=q \omega_{\text {EGAM }} R_{0}$. Time-averages over an EGAM cycle are plotted by thick lines of the same colour. The shadowed region represents the time evolution of the heat flux due to the $E \times B$ drift velocity. (Right) Equilibrium distribution functions without energetic particles (thin dashed line) and with energetic particles just before the excitation of EGAMs using $S_{\mathrm{EP}} / S_{\mathrm{th}}=0.437$ (thick dashed line) and $S_{\mathrm{EP}} / S_{\mathrm{th}}=0.625$ (solid line).

one obtained in the presence of turbulence, while keeping the same radial gradients. This way the simulation is equivalent in terms of EP drive and diamagnetic damping. The time trace of the flux-surface averaged electrostatic potential is plotted in figure 9a in logarithmic scale. The solid red line corresponds to the simulation with turbulence, whereas the thin dashed line corresponds to the simulation without turbulence. Both signals have been plotted using the same time vector, for comparison. It can be observed that in both cases EGAMs are excited exhibiting similar linear growth rates. However, the absence of turbulence leads to an increased nonlinearly saturated amplitude. In addition, we plot the time evolution of the slope of the equilibrium distribution function at the resonant parallel velocity in figure $9 \mathrm{~b}$ and represented by the magenta curve. As in figure 8a, time-averaged quantities over an EGAM cycle are represented by thick lines of the same colour. Several comments can be made based on these figures. First, the reduction of the slope 


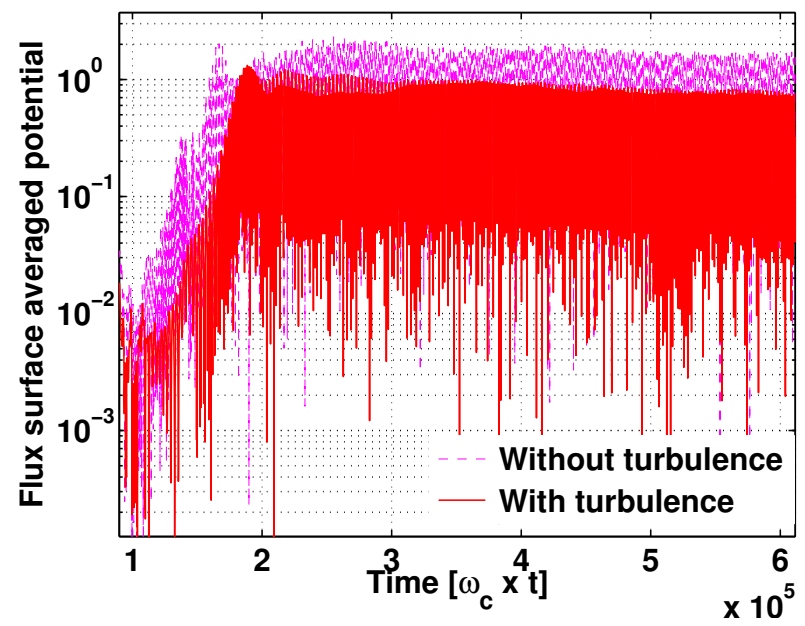

(a)

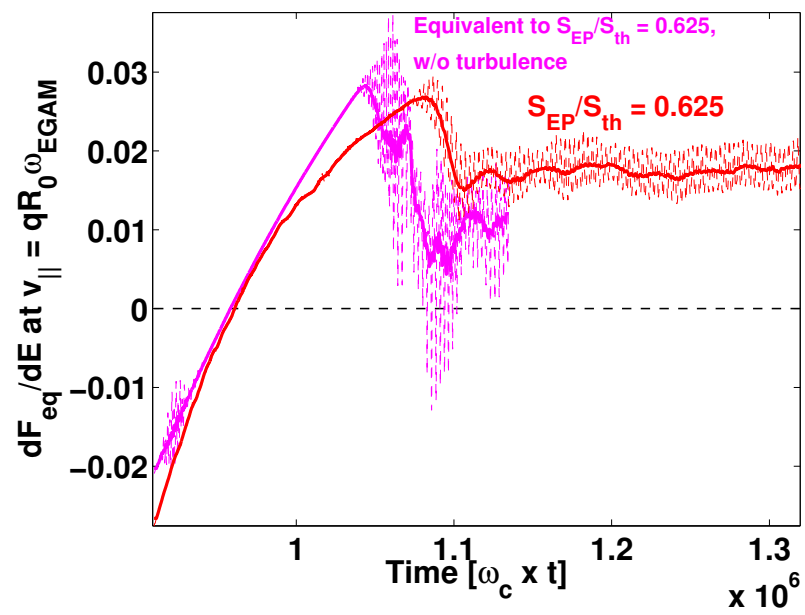

(b)

FIG. 9: (Left) Time evolution of the flux-surface averaged electrostatic potential with (solid red line) and without (dashed magenta line). (Right) Comparison of the slope of the equilibrium distribution function at the resonant velocity with (red line) and without (magenta line) turbulence.

during the nonlinear saturation is more dramatic in the absence of turbulence. Second, the time evolution of the slope evolves smoothly in the presence of turbulence, compared to the situation where turbulence is suppressed, characterised by a more pronounced bursty behaviour. Finally, the amplitude of the oscillations of the distribution function is increased when filtering out the non axisymmetric modes. These observations in the electrostatic potential and the distribution function indicate that in the absence of turbulence kinetic effects play a more pronounced role in the saturation of EGAM, whereas in the presence of turbulence a channel of energy from EGAMs to turbulence is possible.

A second indication of the interaction between ITG modes and EGAMs, is that the nonlinear saturation of the EGAM, visible mainly because of the partial flattening of the distribution function, is accompanied by a sharp increase of the $E \times B$ heat flux (around $\omega_{c} t=1.1 \cdot 10^{6}$ ), which occurs in the same time scale as the nonlinear saturation of the EGAM. This time evolution of the $E \times B$ heat flux indicates a possible nonlinear interaction between ITG modes and EGAMs, which can occur 
nonlocally by means of coupling between outwards propagating fronts linked to ITG modes and static oscillations of radial profiles linked to EGAMs, as invoked in [13]. A local mechanism might also exist by means of a parametric decay, as in the case of standard GAM and ITG modes [25], represented by a resonant three-wave interaction dominating over the wave-particle interaction.

Note that two additional mechanisms could be invoked for the modification and increase of turbulence. First, since the source is depleting the population resonating with ITG modes, one could expect that the nonlinear saturation of EGAMs would redistribute particles in velocity space and therefore feed again the ITG instability. However, as can be observed from figure 5, after the EGAM saturation, the value of the equilibrium distribution function remains below $50 \%$ the initial value (i.e. $\left.\int \mathrm{d} \mu F_{\text {eq }} / \int \mathrm{d} \mu F_{\text {eq }}(t=0)<0.5\right)$. Since the reduction of turbulence occured with a reduction of more than $30 \%$ (i.e. $\int \mathrm{d} \mu F_{\text {eq }} / \int \mathrm{d} \mu F_{\text {eq }}(t=0)<0.7$ ) one can conclude that the redistribution of particles in velocity space during the nonlinear saturation of EGAM is not enough to explain the increase of turbulent intensity. Second, in the calculation of the $E \times B$ heat flux we are considering also the axisymmetric modes. Therefore, one could think that those modes also contribute to the increase of the heat flux. Nevertheless, it was reported in [13] that axisymmetric modes excited by energetic particles do not contribute directly to the turbulent transport estimated by the $E \times B$ heat flux. For these reasons, in the remainder of this paper we look for evidences of a local nonlinear coupling between EGAMs and non axisymmetric modes that could explain the impact of EGAM saturation on turbulence.

Finally, one should note that the abrupt increase of turbulent transport during the nonlinear EGAM saturation leads to a decrease of the temperature gradient, as observed in figure 6b, which occurs just after the nonlinear saturation of EGAM. Further features of EGAMs in the absence of turbulence such as the formation of holes and clumps in velocity space [14], the dependence of the nonlinear saturated state on the drive and the long time evolution of the distribution function of $\mathrm{EP}$ in multi-ion simulations without turbulence using an EP source deserve more attention than the one drawn in the present paper and will therefore be left for a future publication. 


\section{RADIAL STRUCTURE OF EGAM AND MODIFICATION OF TURBULENCE INTENSITY AND TRANSPORT}

The structure of EGAM has been little explored in the previous litterature. EGAM is believed to have a global structure [3] and has been found in simulations with imposed background distribution function to exhibit a structure constrained by the Dirichlet boundary conditions [6], with a frequency that is constant in the whole radial domain, as in experiments $[9,10,26]$. In addition, in the presence of a localised source, the EGAM has been found to be trapped where the drive is strongest with an exponential damping due to the continuous GAM [4]. In our simulations, the source cannot be strongly localised, in order to minimise the direct impact on the radial gradients and therefore on the ITG modes. However, due to the way the EP source is implemented, the EP drive, i.e. the positiveness of $\partial F_{\mathrm{eq}} / \partial E$, does not follow the same radial dependence as the EP source [13]. In that way, we can infere that the effective EP source is localised in a region $0.4<r / a<0.8$ [13], which is equivalent to $60 \rho_{i}<r<120 \rho_{i}$. The radial structure of the EGAM is analysed in this paper from the electrostatic potential $\phi$. We determine the frequency of the mode from the time dependent flux-surface averaged electrostatic potential $\phi_{00}\left(r_{\text {mid }}, t\right)$ evaluated at the mid radial position $r_{\text {mid }}$. Owing to the poloidal and toroidal structures of the EGAM-like potential, we filter out all the non-axisymmetric toroidal components and keep only the $m=1,2$ poloidal components that oscillate at the EGAM frequency. Once the filter applied, the sine and cosine components of the $m=1$ and $m=2$ modes can be obtained. These components are plotted in figure 10 for the simulation with an EP source $S_{\mathrm{EP}} / S_{\text {th }}=0.625$. It can be observed that the $\sin \theta$ component dominates over the $\cos \theta$ component and is mainly localised in the region $60 \rho_{i}<r<120 \rho_{i}$, where the EP drive is strongest. It is also observed that the $\cos \theta, \sin 2 \theta$ and $\cos 2 \theta$ are of the same order, extending slightly radially further in. The localisation of the $\sin \theta$ component results in the localisation of the radial electric field calculated as $E_{r} \approx-\partial \phi_{00} / \partial r$ at the EGAM frequency. The oscillating radial electric field can therefore be able to interact with ITG modes, modulating and modifying this way the amplitude of the turbulent intensity and the $E \times B$ heat flux.

The modification of the radial structure of turbulent intensity is analysed by using the expression 13 evaluated at the end of the simulations within a sufficiently long time window to ensure that the system has reached a statistically steady state. The radial dependence of the turbulent intensity is 


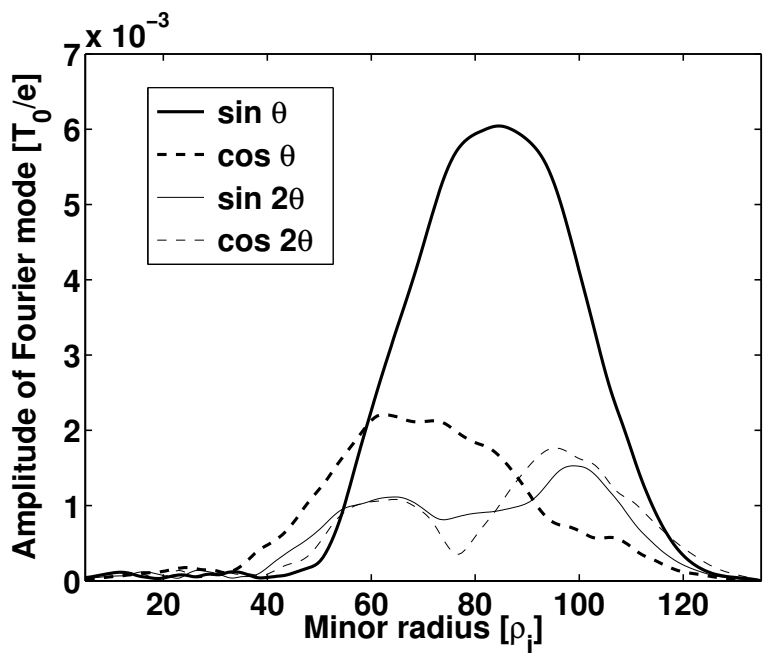

FIG. 10: Radial dependence of the $m=1$ (thick lines) and $m=2$ (thin lines) poloidal components of the electrostatic potential oscillating at the EGAM frequency. The sine and cosine components are evidenced by solid and dashed lines, respectively.

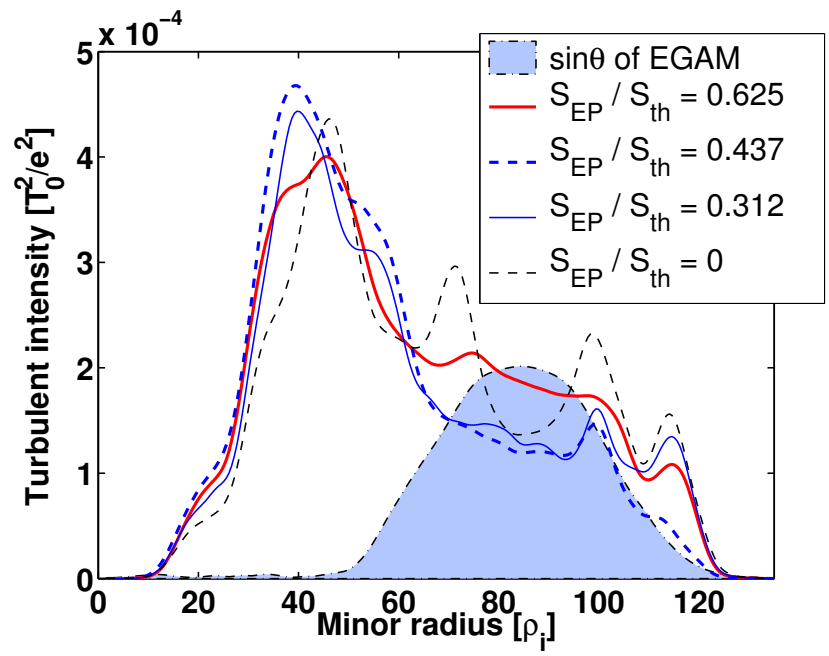

FIG. 11: For the three simulations used in this paper, plotted is the turbulent intensity at the end of the simulations as measured by 13 for a sufficiently long time window where the system exhibits a statistically steady state. For reference, the case without EP source is given (dashed thin line). The shadowed region represents the radial localisation of the EGAM. 
plotted in figure 11, where also the turbulent intensity in the absence of EP is given for reference (thin dashed line), as was done in figure 6a. In addition, we plot the radial dependence of the EGAM (shadowed region). Several comments can be made based on this figure. First, the staircase structure observed in the absence of EP source disappears in the presence of EP. The plasma staircase plays an important role in organising the energy transport, as the long-range radial propagation of energy by means of avalanches is interrupted statistically by avalanche-staircase interplay $[23,24]$. When the staircase structure disappears, energy transport that would be interrupted can expand over a larger radial domain, as observed in [13]. Second, in the region where the EGAM is not excited, the turbulent intensity is close to the one in the absence of EP, for any amplitude of the EP source. Third, the main modification occurs in the region where the EGAM is localised. It is to be noted that the turbulent intensity remains lower than the reference intensity for the two cases where the EGAM amplitude is weaker (blue lines). When the EGAM amplitude is increased (red line), the turbulent intensity is also increased, but remains of the same order as the intensity in the absence of EP. The main quantity to be analysed within the framework of the transport in a tokamak is the radial $E \times B$ heat flux, given by equation 19. In figure 12a, the time-averaged $E \times B$ heat flux over a time window $\omega_{c} \Delta t>10^{5}$ is plotted as a function of the minor radius for the simulations used in this paper. It can be observed that overall the heat flux when the EP drive is stronger is increased with respect to the simulations where the amplitude of the EP source is reduced.

\section{NONLINEAR COUPLING BETWEEN EGAMS AND ITG MODES}

Note that in figure $12 \mathrm{a}$ we plot time-averaged quantities. However, Fourier analysis shows that the $E \times B$ heat flux oscillates at the EGAM frequency. The amplitude of the Fourier mode is represented in figure $12 \mathrm{~b}$ as a function of the frequency. The dominant peak corresponds to the EGAM frequency. For completeness, the first and second harmonics are also included in the figure. Since the $E \times B$ heat flux is a nonlinear quantity, those oscillations must be due to the nonlinear interaction between modes satisfying the matching conditions $\omega_{1}+\omega_{2}=\omega_{\mathrm{EGAM}}$ and $\mathbf{k}_{1}+\mathbf{k}_{2}=\mathbf{k}_{\mathrm{EGAM}}$. The EGAM is mainly represented by the mode $\phi_{00}$ oscillating at the EGAM frequency. Therefore, we need to look for the possibility to have non axisymmetric modes with 


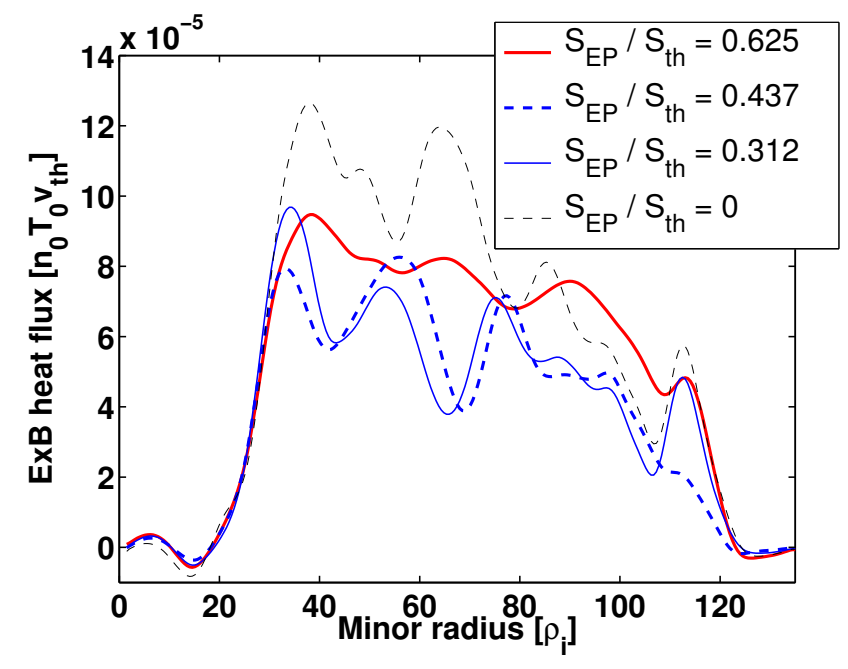

(a)

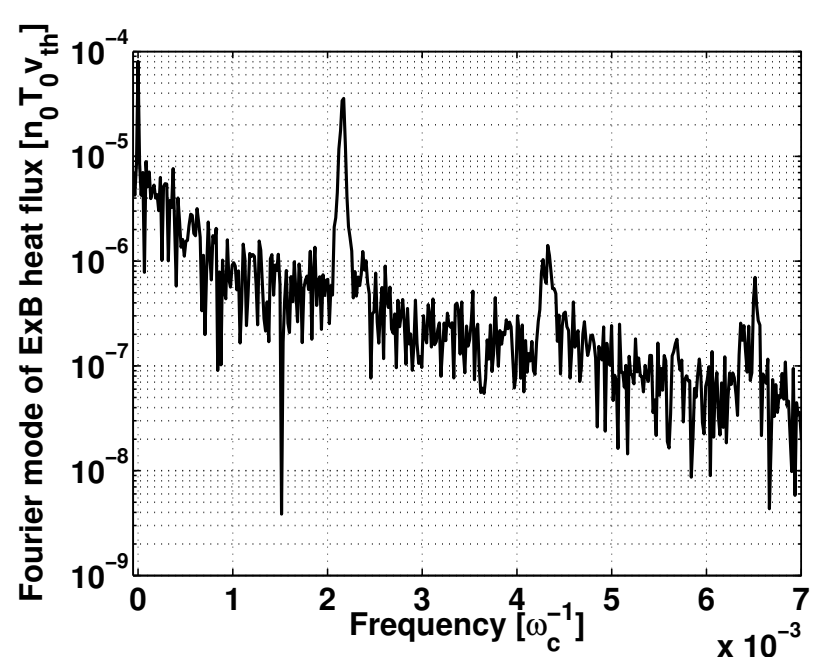

(b)

FIG. 12: (Left) Radial dependence of the time-averaged $E \times B$ heat flux for the simulations of this paper. For comparison, the heat flux in the absence of EP is also plotted and represented by the thin dashed black line. (Right) Time Fourier transform of the $E \times B$ heat flux evaluated at the mid radial position for the simulation with $S_{\mathrm{EP}} / S_{\mathrm{th}}=0.625$.

opposite poloidal and toroidal mode numbers and whose frequencies sum up the EGAM frequency. This has been by means of a bispectral analysis of the toroidal and poloidal Fourier modes of the electrostatic potential at the mid radial position, called in the following $\hat{\phi}_{m, n}$. The bispectral analysis used in this paper is based on a wavelet decomposition in time as was done in [27] and explained in previous works [28, 29]. For this purpose a Morlet wavelet function is chosen, having the following expression

$$
\psi_{\omega}(t)=\sqrt{\frac{\omega}{2 \pi}} \mathrm{e}^{i \omega t} \mathrm{e}^{-\frac{1}{2}\left(\frac{\omega t}{2 \pi}\right)^{2}}
$$

where $\omega \in \mathbb{R}$ is the frequency representing here the wavelet scale. The wavelet transform of the electrostatic potential $\hat{\phi}_{m, n}$, similarly to the Fourier transform, is defined as

$$
W_{\hat{\phi}_{m, n}}(\omega, t)=\int_{\mathbb{R}} \hat{\phi}_{m, n}(\tau) \psi_{\omega}(\tau-t) \mathrm{d} \tau
$$

The wavelet transform is calculated considering that it is a convolution product. Therefore, the Fourier transform of the wavelet transform is related to the Fourier transforms of the electrostatic 
potential and the Morlet wavelet function as $\mathcal{F}\left[W_{\hat{\phi}_{m, n}}\right]=\mathcal{F}\left[\hat{\phi}_{m, n}\right] \mathcal{F}\left[\psi_{\omega}\right]$, with $\mathcal{F}[\cdot]$ the Fourier transform. Then, the wavelet transform simply reads $W_{\hat{\phi}_{m, n}}=\mathcal{F}^{-1}\left[\mathcal{F}\left[\hat{\phi}_{m, n}\right] \mathcal{F}\left[\psi_{\omega}\right]\right]$, where $\mathcal{F}^{-1}[\cdot]$ is the inverse Fourier transform. In this paper we restrict the analysis to the interaction between $\hat{\phi}_{0,0}$ and all the possible poloidal and toroidal modes $\left(m_{1}, n_{1}\right),\left(m_{2}, n_{2}\right)$ satisfying $m_{1}+m_{2}=0$ and $n_{1}+n_{2}=0$. This is reflected by the definition of the wavelet bispectrum

$$
b^{w}\left(\omega_{1}, \omega_{2}\right)=\int \mathrm{d} t\left(W_{\hat{\phi}_{0,0}}(\omega, t)\right)^{\dagger} \sum_{m, n} W_{\hat{\phi}_{m, n}}\left(\omega_{1}, t\right) W_{\hat{\phi}_{-m,-n}}\left(\omega_{2}, t\right)
$$

where the integral is in practice performed over a finite time interval, $W^{\dagger}$ represents the complex conjugate of the quantity $W$ and the frequencies $\omega_{1}$ and $\omega_{2}$ satisfy the matching condition $\omega_{1}+\omega_{2}=$ $\omega$. If a nonlinear interaction exists between two waves of frequencies $\omega_{1}$ and $\omega_{2}$ and a third one of frequency $\omega$ this must be observed by the presence of straight lines intersecting the vertical axis at the frequency $\omega$.

The analysis has been performed only for the simulation with the largest amplitude of energetic particle source. Four time windows are analysed. They correspond to (1) the phase where turbulence is not reduced yet, (2) the linear excitation of EGAMs until the end of the nonlinear saturation, (3) the phase just after the nonlinear saturation of EGAMs and the increase of turbulence and (4) the steady-state phase where EGAMs and turbulence coexist. A structure characterised by a straight line crossing the point $\left(\omega_{1}=0, \omega_{2}=0\right)$ is clearly observed before the onset of EGAMs (figure 13a), meaning that the main interaction occurs between non axisymmetric modes and the zonal structure oscillating at $\omega \approx 0$, which is the well-known mechanism of self-regulation of turbulence by stationary zonal flows [20, 30, 31]. During the linear excitation and nonlinear saturation of EGAMs (figure 13b), the same structure characterising the nonlinear interaction between ITG modes and zonal flows is still present, together with a second one characterising the nonlinear interaction between non axisymmetric modes oscillating at $\omega_{1}$ and $\omega_{2}$ and a zonal component oscillating at a frequency $\omega \neq 0$. This non-zero frequency is evidenced by the analysis of the wavelet bispectrum at later times, as shown in the next figures. In particular, just after the saturation of EGAMs (figure 13c), two nonlinear interactions are clearly observed: one is the standard interaction between non axisymmetric modes and zero frequency zonal modes and the other one is the nonlinear interaction between non axisymmetric modes and zonal components at EGAM frequency (highlighted by two horizontal yellow dashed lines). When analysing the non- 


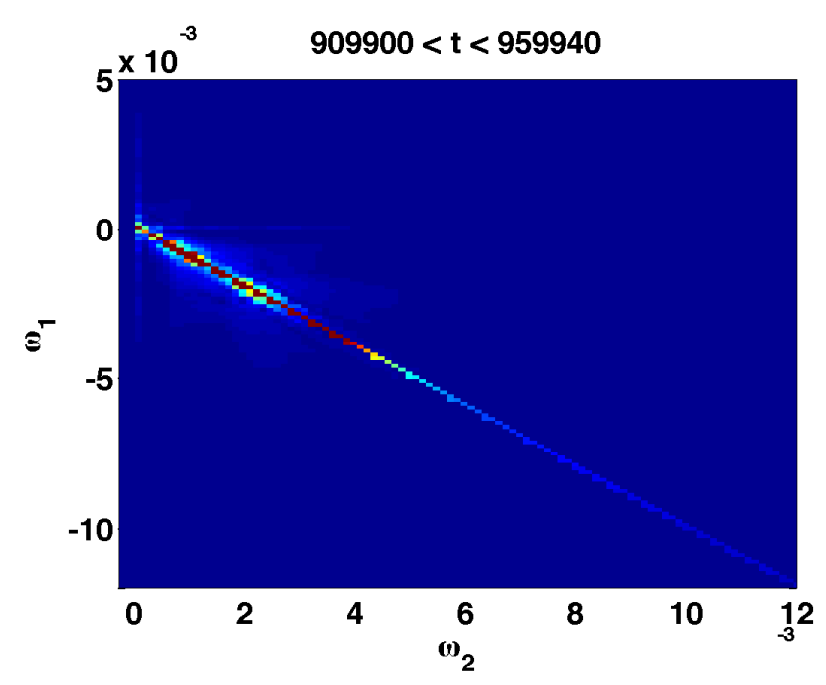

(a)

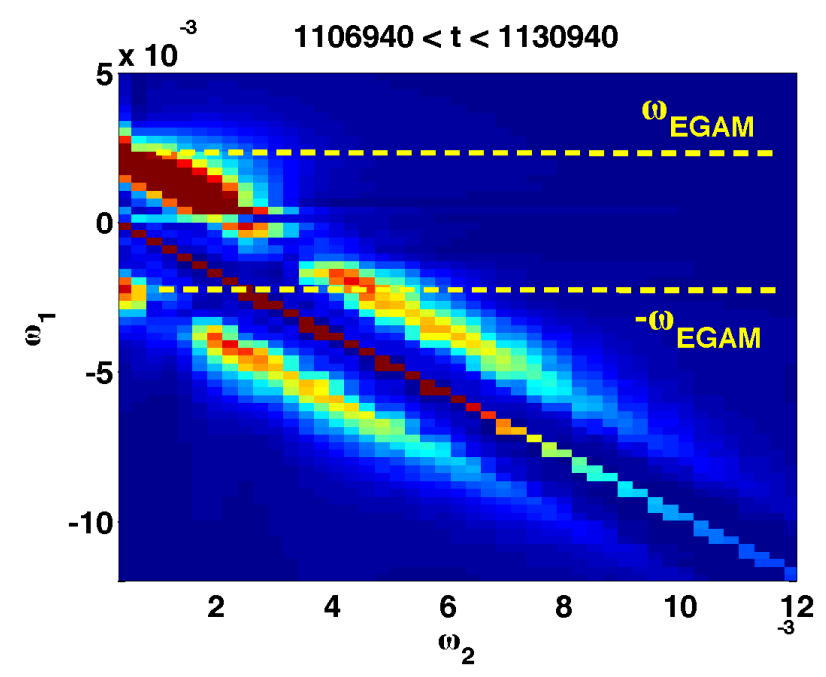

(c)

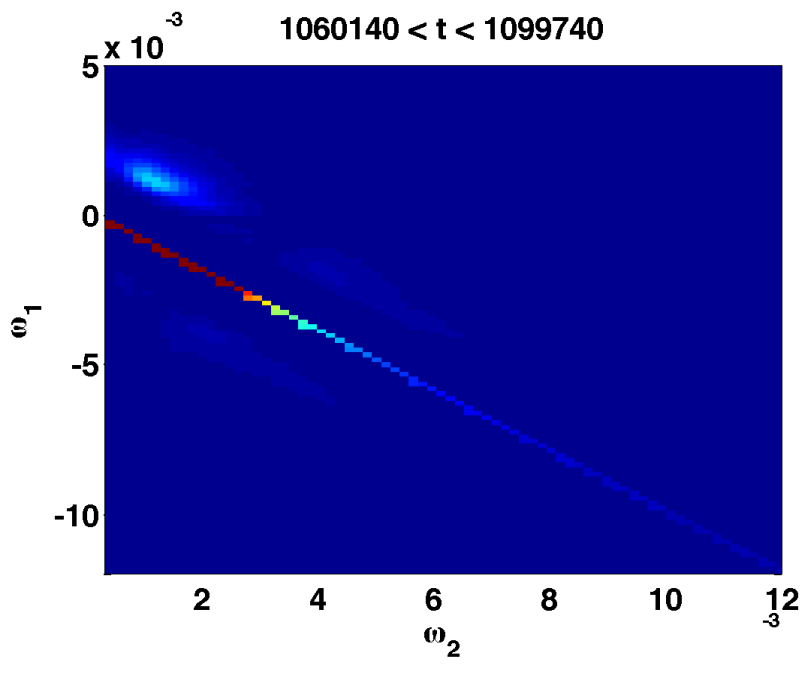

(b)

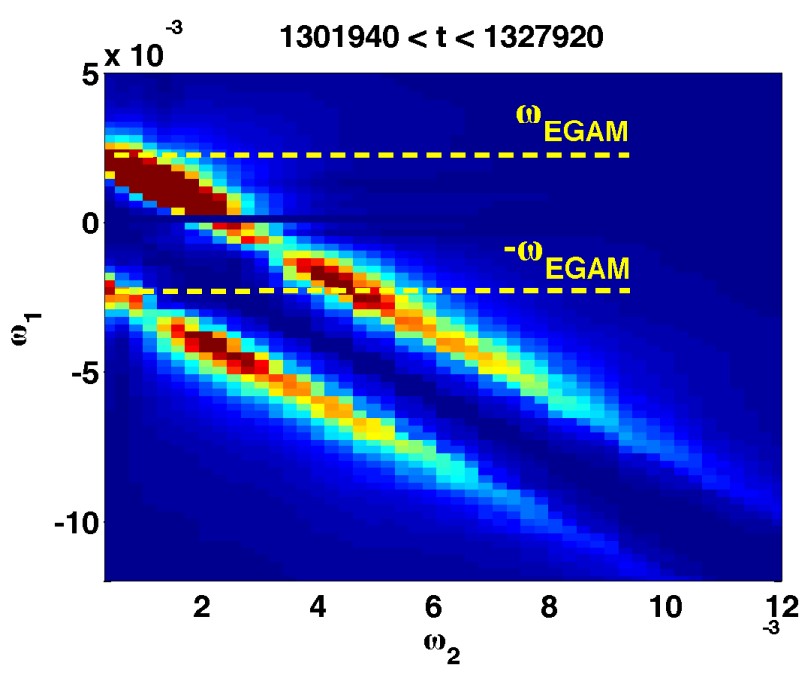

(d)

FIG. 13: Wavelet bispectrum for the four phases indicated in the main text of the paper and on top of each of the subfigures. For clarity and only in the last two figures, the EGAM frequency has been indicated by horizontal yellow dashed lines.

linear interaction during the steady-state (figure 13d), the standard self-regulation of turbulence by zonal flows that should be visible by the straight line crossing the point $\left(\omega_{1}=0, \omega_{2}=0\right)$ disappears and the dynamics is completely dominated by the nonlinear interaction between three waves 
satisfying $\omega_{1}+\omega_{2}=\omega_{\text {EGAM }}$. It is to be noted that analysis of different energy channels during the nonlinear interaction between turbulence and zonal flows was reported in the past using a global electromagnetic Landau fluid code [30, 31], evidencing that a part of the energy of the oscillatory zonal flows (GAMs) is transferred back to the turbulence via the poloidally asymmetric component of the pressure perturbation. This was the case for nonlinearly self-generated zonal flows in the absence of energetic particles. However, in the present paper, the generation of oscillatory zonal flows is done by means of energetic particles. Moreover, all the modification of the distribution function by the energetic particle source is essential for the initial reduction of turbulence, as reported in [13] and explained in section III. Therefore, although the results presented in this section are based on a three-wave interaction of the electrostatic potential as could be done in fluid codes, the gyro-kinetic approach reveals essential to evidence the energy transfer from energetic particles to turbulence via EGAMs.

\section{CONCLUSIONS AND FUTURE WORK}

In this paper we have presented full- $f$ global gyro-kinetic simulations of energetic-particle-driven geodesic acoustic modes in the presence of turbulence using the GysELA code. For the first time, the radial structure of the EGAM in global simulations with realistic radial profiles is reported, showing that the mode is localised in a radial region where the EP drive is strongest. A scan on the amplitude of the EP source has been performed, showing that the drive of energetic particles increases with the amplitude of the source and so is the amplitude of the EGAM. It has also been reported that the $E \times B$ heat flux is increased during the nonlinear saturation of the EGAM, suggesting a transfer of energy from EGAM to turbulent modes. This transfer has been evidenced by comparing the turbulent simulations with an equivalent simulation from the point of view of the EP drive but without turbulence, showing that the flattening of the distribution function in velocity space is more pronounced in the absence of turbulence and the amplitude of the electrostatic potential is increased. This indicates that, in the absence of turbulence, kinetic effects via waveparticle interaction play a more important role in the nonlinear saturation of EGAMs. However, background turbulence acts as a sink of energy via three-wave interaction between non axisymmetric modes and EGAMs. Bispectral analysis using Fourier transform in poloidal and toroidal directions 
and wavelet transform in time shows a clear nonlinear interaction between non axisymmetric modes

and zonal structures. This interaction evolves from the standard self-regulation of turbulence by the zero-frequency zonal component to a steady-state regime where turbulence dynamics is dominated by the zonal component oscillating at the EGAM frequency. The nonlinear three-wave coupling between EGAMs and turbulence is likely to provide a saturation mechanism that adds to the standard wave-particle interaction increasing this way the amplitude of axisymmetric modes that contribute to the transport of energy and particles, modulated at the EGAM frequency.

In the present work we have used a simplified model for the energetic particle source and focus our analysis on the nonlinear interaction between EGAMs and turbulent modes. However, further studies are required to shed light on the direct impact of realistic energetic particle sources on turbulence and confinement properties. In addition, detailed statistical analysis to determine the intermittent and bursty behaviour of turbulence together with the modification of turbulent and neoclassical transports in the presence of energetic particles is needed for completeness and will be included in a future publication. Finally, our work has been done in the electrostatic limit using EGAMs. However, EGAMs can be understood as a paradigm of energetic particle modes whose interaction with turbulence can be observed in simplified models like the one used in this paper. The interaction EGAM-turbulence should be extended to electromagnetic regimes where Alfvén modes can interact with electromagnetic turbulence.

\section{ACKNOWLEDGMENTS}

This work has been carried out within the framework of the EUROfusion Consortium and has received funding from the European Union's Horizon 2020 research and innovation programme under grant agreement number 633053 and from the A*MIDEX project (no. ANR-11-IDEX-000102) funded by the "Investissements d'Avenir" French Government program, managed by the French National Research Agency (ANR). The views and opinions expressed herein do not necessarily reflect those of the European Commission. Simulations were performed on the IFERC-CSC Helios supercomputer within the framework of the TURBEP project. This work has been done within the framework of the nonlinear energetic particle dynamics (NLED) European Enabling Research Project (EUROFUSION WP15-ER-01/ENEA-03). The authors acknowledge fruitful discussions 
with Y. Sarazin, X. Garbet and G. Dif-Pradalier.

[1] Philipp Lauber. Physics Reports, 533(2):33-68, 2013.

[2] Liu Chen and Fulvio Zonca. Reviews of Modern Physics, 88(1):015008, 2016.

[3] GY Fu. Physical review letters, 101(18):185002, 2008.

[4] Z. Qiu, F. Zonca, and L. Chen. Plasma Physics and Controlled Fusion, 52:095003, 2010.

[5] Z. Qiu and L. Chen. Plasma Science and Technology, 13:257, 2011.

[6] D. Zarzoso, X. Garbet, Y. Sarazin, R. Dumont, and V. Grandgirard. Physics of Plasmas, 19(2):022102-022102, 2012.

[7] D Zarzoso, A Biancalani, A Bottino, Ph Lauber, E Poli, J-B Girardo, X Garbet, and RJ Dumont. Nuclear Fusion, 54(10):103006, 2014.

[8] Jean-Baptiste Girardo, David Zarzoso, Rémi Dumont, Xavier Garbet, Yanick Sarazin, and Sergei Sharapov. Physics of Plasmas (1994-present), 21(9):092507, 2014.

[9] HL Berk, CJ Boswell, D Borba, ACA Figueiredo, T Johnson, MFF Nave, SD Pinches, SE Sharapov, et al. Explanation of the jet $\mathrm{n}=0$ chirping mode. Nuclear fusion, 46(10):S888, 2006.

[10] CJ Boswell, HL Berk, DN Borba, T. Johnson, SD Pinches, and SE Sharapov. Physics Letters A, $358(2): 154-158,2006$.

[11] R. Nazikian, GY Fu, ME Austin, HL Berk, RV Budny, NN Gorelenkov, WW Heidbrink, CT Holcomb, GJ Kramer, GR McKee, et al. Physical review letters, 101(18):185001, 2008.

[12] HL Berk and T. Zhou. Nuclear Fusion, 50:035007, 2010.

[13] D Zarzoso, Y Sarazin, X Garbet, R Dumont, A Strugarek, J Abiteboul, T Cartier-Michaud, G DifPradalier, Ph Ghendrih, V Grandgirard, et al. Physical Review Letters, 110(12):125002, 2013.

[14] Hao Wang, Yasushi Todo, and Charlson C. Kim. Phys. Rev. Lett., 110:155006, Apr 2013.

[15] Alessandro Biancalani, Alberto Bottino, Ph Lauber, and David Zarzoso. Nuclear Fusion, 54(10):104004, 2014.

[16] Hao Wang, Yasushi Todo, Takeshi Ido, and Masaki Osakabe. Physics of Plasmas, 22(9), 2015. 
[17] N. Winsor, J.L. Johnson, and J.M. Dawson. Physics of Fluids, 11:2448, 1968.

[18] K. Hallatschek and D. Biskamp. Physical Review Letters, 86(7):1223-1226, 2001.

[19] GD Conway, C. Angioni, F. Ryter, P. Sauter, and J. Vicente. Physical Review Letters, 106(6):65001, 2011.

[20] PH Diamond, SI Itoh, K. Itoh, and TS Hahm. Plasma Physics and Controlled Fusion, 47:R35, 2005.

[21] V. Grandgirard, J. Abiteboul, J. Bigot, T. Cartier-Michaud, N. Crouseilles, G. Dif-Pradalier, Ch. Ehrlacher, D. Esteve, X. Garbet, Ph. Ghendrih, G. Latu, M. Mehrenberger, C. Norscini, Ch. Passeron, F. Rozar, Y. Sarazin, E. Sonnendrcker, A. Strugarek, and D. Zarzoso. Computer Physics Communications, 207:35 - 68, 2016 .

[22] AJ Brizard and TS Hahm. Foundations of nonlinear gyrokinetic theory. Reviews of modern physics, $79(2): 421,2007$.

[23] G Dif-Pradalier, PH Diamond, V Grandgirard, Y Sarazin, J Abiteboul, X Garbet, Ph Ghendrih, A Strugarek, S Ku, and CS Chang. Physical Review E, 82(2):025401, 2010.

[24] G Dif-Pradalier, Grégoire Hornung, Ph Ghendrih, Y Sarazin, F Clairet, L Vermare, PH Diamond, J Abiteboul, T Cartier-Michaud, C Ehrlacher, et al. Physical review letters, 114(8):085004, 2015.

[25] F. Zonca and L. Chen. EPL (Europhysics Letters), 83:35001, 2008.

[26] W Chen, XT Ding, LM Yu, XQ Ji, ZB Shi, YP Zhang, WL Zhong, GL Yuan, JQ Dong, and QW Yang. Observation of energetic-particle-induced gam and nonlinear interactions between egam, baes and tearing modes on the hl-2a tokamak. Nuclear Fusion, 53(11):113010, 2013.

[27] B Ph Van Milligen, C Hidalgo, and E Sanchez. Nonlinear phenomena and intermittency in plasma turbulence. Physical review letters, 74(3):395, 1995.

[28] Charles Meneveau. Analysis of turbulence in the orthonormal wavelet representation. Journal of Fluid Mechanics, 232:469-520, 1991.

[29] Lonnie Hudgins, Carl A Friehe, and Meinhard E Mayer. Wavelet transforms and atmopsheric turbulence. Physical Review Letters, 71(20):3279, 1993.

[30] Naoaki Miyato, Yasuaki Kishimoto, and Jiquan Li. Global structure of zonal flow and electromagnetic ion temperature gradient driven turbulence in tokamak plasmas. Physics of plasmas, 11(12):55575564, 2004. 
[31] N Miyato, JQ Li, and Y Kishimoto. Study of a drift wave-zonal mode system based on global electromagnetic landau-fluid itg simulation in toroidal plasmas. Nuclear fusion, 45(6):425, 2005. 